\title{
Targeting treatable traits in severe asthma: a randomised controlled trial
}

\author{
Vanessa M. McDonald (101,2,3,4,5 , Vanessa L. Clark ${ }^{1,2,3,5}$, Laura Cordova-Rivera ${ }^{1,2,3}$, \\ Peter A.B. Wark (10) ${ }^{1,2,3,4}$, Katherine J. Baines ${ }^{1,2,3}$ and Peter G. Gibson (1) ${ }^{1,2,3,4}$
}

Affiliations: ${ }^{1}$ National Health and Medical Research Council, Centre of Excellence in Severe Asthma, Newcastle, Australia. ${ }^{2}$ Priority Research Centre for Healthy Lungs, The University of Newcastle, Newcastle, Australia. ${ }^{3}$ Hunter Medical Research Institute, Newcastle, Australia. ${ }^{4}$ Dept of Respiratory and Sleep Medicine, John Hunter Hospital, Newcastle, Australia. ${ }^{5}$ School of Nursing and Midwifery, University of Newcastle, Newcastle, Australia.

Correspondence: Vanessa M. McDonald, Level 2 West Wing, 1 Kookaburra Circuit, New Lambton Heights, NSW 2305, Australia. E-mail: Vanessa.McDonaldanewcastle.edu.au

@ERSpublications

Treatable traits is a new paradigm for the management of airway disease. Managing patients with severe asthma using this approach improves health-related quality of life, and validates this as a useful management paradigm for severe asthma. http://bit.ly/2R9xRJh

Cite this article as: McDonald VM, Clark VL, Cordova-Rivera L, et al. Targeting treatable traits in severe asthma: a randomised controlled trial. Eur Respir J 2020; 55: 1901509 [https://doi.org/10.1183/ 13993003.01509-2019].

\section{ABSTRACT}

Rationale: Treatable traits have been proposed as a new paradigm for airway disease management.

Objectives: To characterise treatable traits in a severe asthma population and to determine the efficacy of targeting treatments to these treatable traits in severe asthma.

Methods: Participants $(\mathrm{n}=140)$ with severe asthma were recruited to a cross-sectional study and underwent a multidimensional assessment to characterise treatable traits. Eligible participants with severe asthma $(n=55)$ participated in a 16-week parallel-group randomised controlled trial to determine the feasibility and efficacy of management targeted to predefined treatable traits, compared to usual care in a severe asthma clinic. The patient-reported outcome of health-related quality of life was the trial's primary end-point.

Main results: Participants with severe asthma had a mean \pm SD of $10.44 \pm 3.03$ traits per person, comprising $3.01 \pm 1.54$ pulmonary and $4.85 \pm 1.86$ extrapulmonary traits and $2.58 \pm 1.31$ behavioural/risk factors. Individualised treatment that targeted the traits was feasible and led to significantly improved health-related quality of life ( 0.86 units, $\mathrm{p}<0.001)$ and asthma control $(0.73, \mathrm{p}=0.01)$.

Conclusions: Multidimensional assessment enables detection of treatable traits and identifies a significant trait burden in severe asthma. Targeting these treatable traits using a personalised-medicine approach in severe asthma leads to improvements in health-related quality of life, asthma control and reduced primary care acute visits. Treatable traits may be an effective way to address the complexity of severe asthma.

This article has an editorial commentary: https://doi.org/10.1183/13993003.00002-2020

This study is registered as a clinical trial with the Australia New Zealand Clinical Trials Register (ACTRN12612000807853). Individual participant data will be available upon request, after de-identification. The protocol will also be made available. Data will be made available from 9 months until 36 months following article publication. Data sharing will occur with investigators whose proposed use of the data has been approved by an independent review committee identified for this purpose; proposals may be submitted up to 36 months following article publication. Data will be available for individual patient meta-analysis. After 36 months the data will be available in our university's data warehouse, but without investigator support other than the deposit of metadata.

This article has supplementary material available from erj.ersjournals.com

Received: 30 July 2019 | Accepted after revision: 23 Nov 2019

Copyright OERS 2020 


\section{Introduction}

Severe asthma is a complex and heterogeneous airway disease characterised by multiple aggravating factors, several clinical phenotypes and biological endotypes and frequent comorbidities [1, 2]. While asthma is common, with an estimated global prevalence of 334 million people [3], severe asthma is less prevalent, affecting $\sim 3 \%$ of this group $[1,4]$. Despite this, the burden of disease from severe asthma is excessive [5], from patient [6-8], healthcare and economic perspectives [9, 10].

The stepped-treatment approach that is effective in the management of mild-to-moderate asthma fails people with severe disease due to their treatment refractoriness and the condition's heterogeneous nature [11]. In response to this and the stagnation of asthma outcomes in some countries [12], there are calls for new approaches to severe asthma that involve the application of personalised medicine, which deconstructs airway disease into its components, and applies individualised management according to recognised traits $[5,11,13]$. Specific examples include the treatable traits approach proposed by AGUsti et al. [13], and multidimensional assessment $[14,15]$ approaches where traits are assessed in the pulmonary, extrapulmonary and behavioural/lifestyle risk factor domains and individual treatments are applied according to the identified traits [13].

In a systematic review, several uncontrolled studies suggest a positive effect of multidimensional assessment in severe asthma [16]. We have adapted and expanded this approach to the assessment of severe airway disease by focusing on those treatable traits that are relevant to complex airway disease, including biomarkers to assess known endotypes amenable to a management strategy [14, 15]. We have tested this approach in a proof-of-concept study in chronic obstructive pulmonary disease (COPD) showing significant improvements in health status [17]. While this approach is recommended by the scientific community $[5,13]$, to date there are no randomised controlled trials (RCTs) to assess the efficacy of a treatable traits approach in severe asthma [16].

Therefore, the aims of this study were to apply multidimensional assessment in severe asthma to define the number and type of traits present, and to use an RCT to evaluate the effect of multidimensional assessment and targeted management in severe asthma. We hypothesised that patients with severe asthma would have multiple treatment components to their illness and that treatment targeted to those identified traits would improve health-related quality of life (HRQoL).

\section{Materials and methods}

\section{Study design}

Participants with severe asthma $(\mathrm{n}=140)$ were recruited to a cross-sectional study to undertake a multidimensional assessment to determine the prevalence of treatable traits. The assessment was conducted over two visits; traits were characterised in the pulmonary, extrapulmonary and risk factor/ behavioural domains. The multidimensional assessment has been described elsewhere [14] and is detailed in the supplementary material. Detailed eligibility criteria are listed in the supplementary material.

Concurrently, eligible participants $(n=55)$ were consecutively recruited to a parallel-group RCT to determine the efficacy of multidimensional assessment and targeted management in comparison to usual care in a severe asthma clinic [18] (figure 1). Participants in both the cross-sectional study and RCT were recruited from the tertiary care respiratory clinics of the John Hunter Hospital (Newcastle, Australia) and through the research database of the Department of Respiratory and Sleep Medicine at John Hunter Hospital.

Ethics approval was received from the Hunter New England human research ethics committee and the University of Newcastle ethics committee (08/08/20/3.10; H-2009-0013). All participants gave written informed consent. The trial was registered on the Australia New Zealand Clinical Trials Register (ACTRN12612000807853).

Concealed random allocation was employed. In order to ensure optimal matching of groups, the process of minimisation [19] was used by a third party to randomise participants into groups. The criteria used for minimisation included the baseline sputum eosinophil and neutrophil counts, and baseline high-sensitivity C-reactive protein (hs-CRP). Outcomes were assessed at 16 weeks (figure 2). Participant and clinician blinding was not possible, due to the nature of the intervention.

\section{Intervention}

The intervention consisted of a 16-week treatment programme of multidimensional assessment and targeted treatment delivered by a case manager. The key intervention components were as follows. 1) Multidimensional assessment: participants underwent assessments within the pulmonary, extrapulmonary and risk-factor/behaviour domains. At completion of the assessment the results were summarised and the identified traits collated. A full description of the multidimensional assessment and 


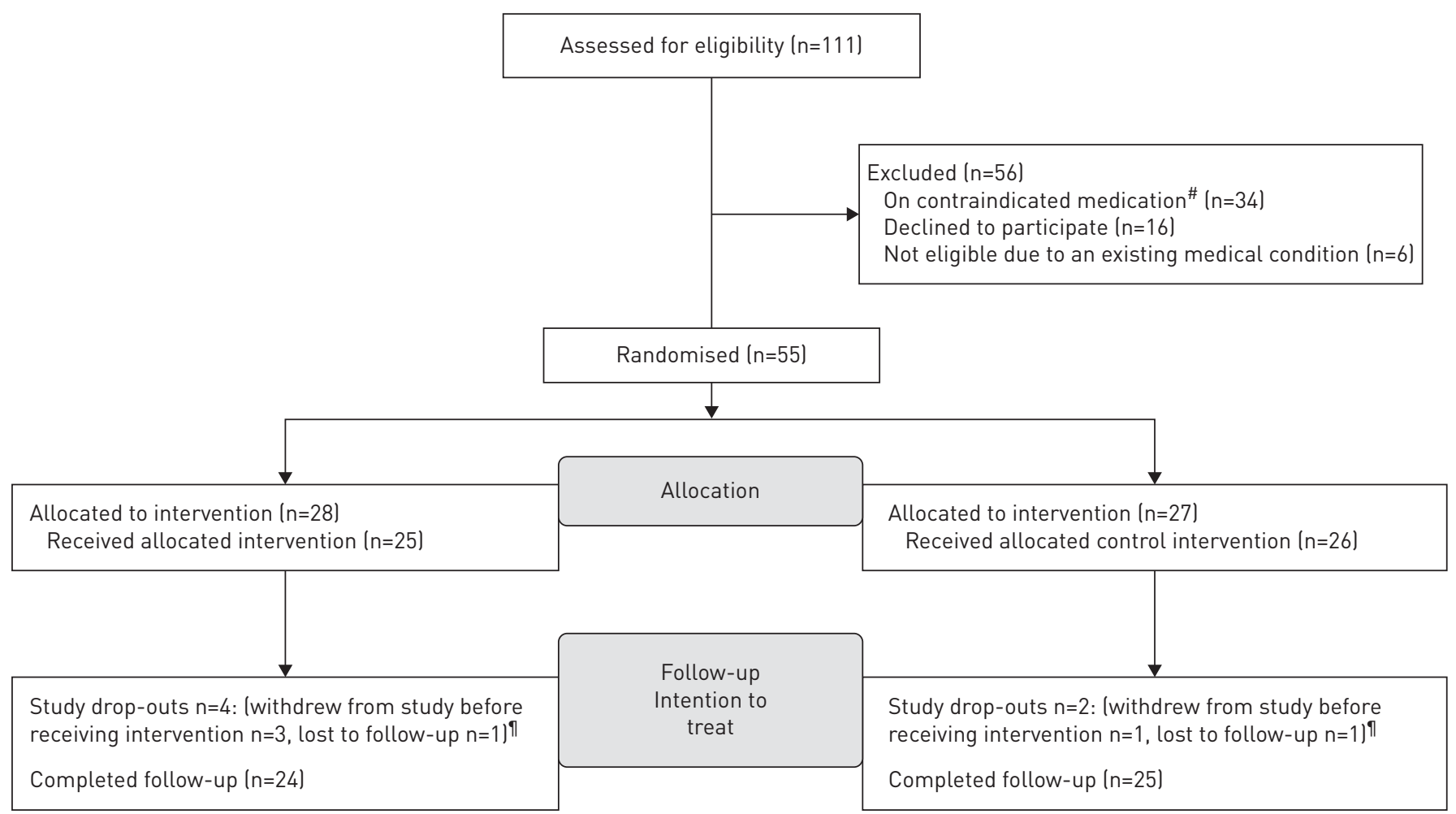

FIGURE 1 CONSORT (Consolidated Standards of Reporting Trials) flow of study participants for the randomised controlled trial. * : contraindicated medication such as omalizumab or a statin; ${ }^{9}$ : included in the analysis as per the intention-to-treat protocol.

associated treatments are presented in table 1 and supplementary table S2, and have been described previously $[14,15]$. 2) Biomarker-driven inflammometry treatment-decision algorithm to identify inflammatory phenotypes and then direct treatment (supplementary table S3). 3) Multidisciplinary treatment plan based on the results of the multidimensional assessment, involving individualised interventions targeted to the identified traits. An example plan is presented in supplementary table S4. 4) Coordination of the packaged interventions by a respiratory nurse case manager (supplementary table S4 and figure 3). The case manager summarised the traits identified, coordinated the case discussion with the multidisciplinary team, communicated the list of traits identified with individual participants, assessed the importance of each trait from the perspective of the physican and the patient, communicated the plan to the patient, provided education regarding the traits and individualised treatments, coordinated the visits between the members of the multidisciplinary team and delivered the interventions for individualised self-management, breath-retraining, cognitive behaviour therapy and smoking cessation. The role of the case manager and discussion with patients has been described previously [20]. Other members of the team included a physican, dietitian, physiotherapist and speech pathologist.

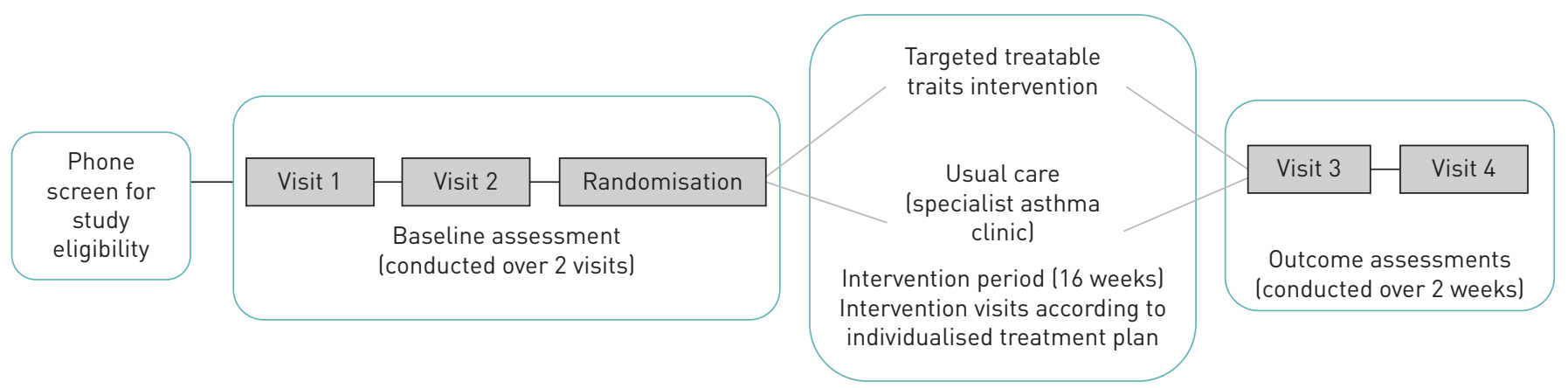

FIGURE 2 Study schema: after screening, participants entered the study for baseline assessment over two visits. Randomisation occurred after the second baseline visit. Follow-up occurred 16 weeks after the baseline second visit. 


\section{TABLE 1 Multidimensional assessment of traits and trait identification markers}

\begin{tabular}{|c|c|}
\hline Trait & Trait identification marker \\
\hline \multicolumn{2}{|l|}{ Pulmonary } \\
\hline Airflow limitation & $\mathrm{FEV}_{1} / \mathrm{FVC}$ ratio $<70 \%$ and $\mathrm{FEV}_{1}<80 \%$ predicted \\
\hline $\begin{array}{l}\text { Airway inflammation } \\
\text { (eosinophilic) }\end{array}$ & Sputum eosinophils $\geqslant 3 \%$ \\
\hline $\begin{array}{l}\text { Airway inflammation } \\
\text { (neutrophilic) }\end{array}$ & Sputum neutrophils $\geqslant 61 \%$ \\
\hline Frequent chest infection & $\geqslant 2$ courses of antibiotics for an exacerbation of chest symptoms \\
\hline Pathogen colonisation & Presence of a recognised bacterial pathogen in sputum \\
\hline Mucus hypersecretion & $\begin{array}{l}\text { Volume } \geqslant 25 \mathrm{~mL} \text { of mucus produced daily for the past week in the } \\
\text { absence of an infection }\end{array}$ \\
\hline Oxygen desaturation & Oxygen saturation levels of $<90 \%$ during $6 \mathrm{MWT}$ \\
\hline Dyspnoea & Dyspnoea score $\geqslant 2$, modified Medical Research Council scale \\
\hline $\begin{array}{l}\text { Systemic allergic } \\
\text { inflammation }\end{array}$ & $\begin{array}{l}\text { Serum IgE level } \geqslant 76 \mathrm{IU} \cdot \mathrm{mL}^{-1} \text { (prescribed according to PBS guidelines } \\
\quad \text { (supplementary table } \mathrm{S} 1 \text { ) }\end{array}$ \\
\hline \multicolumn{2}{|r|}{ 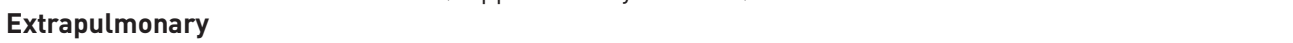 } \\
\hline Dysfunctional breathing & Nijmegen score $\geqslant 23$ \\
\hline Anaemia & $\mathrm{Hb}<120 \mathrm{~g} \cdot \mathrm{L}^{-1}$ for females, $<140 \mathrm{~g} \cdot \mathrm{L}^{-1}$ for males \\
\hline Systemic inflammation & $\mathrm{hsCRP} \geqslant 3 \mathrm{mg} \cdot \mathrm{L}^{-1}$ \\
\hline Daytime sleepiness & Epworth sleep score $>8$ \\
\hline Obesity & $\mathrm{BMI} \geqslant 30 \mathrm{~kg} \cdot \mathrm{m}^{-2}$ \\
\hline Depression and/or anxiety & $\begin{array}{l}\text { Hospital Anxiety and Depression scale ( } \geqslant 8 \text { on anxiety and/or depression } \\
\text { subscale) }\end{array}$ \\
\hline Significant medical history & Patient self-reported other medical conditions \\
\hline Cardiac history & Patient self-reported cardiac conditions \\
\hline Vocal cord dysfunction & $\begin{array}{l}\text { Vocal cord dysfunction questionnaire: total score } \geqslant 5 \text { is positive for } \\
\text { laryngeal dysfunction }\end{array}$ \\
\hline \multicolumn{2}{|l|}{$\begin{array}{l}\text { Risk factors and behavioural } \\
\text { traits }\end{array}$} \\
\hline $\begin{array}{l}\text { Absence of written action } \\
\text { plan }\end{array}$ & $\begin{array}{l}\text { Patient does not possess written action plan or does not use the } \\
\text { prescribed plan during exacerbations }\end{array}$ \\
\hline Exercise tolerance & Distance of $<350 \mathrm{~m}$ on $6 \mathrm{MWT}$ \\
\hline \multicolumn{2}{|l|}{ Bone density } \\
\hline Osteopenia & T-score between $-1.0--2.5$ \\
\hline Osteoporosis & T-score $\leqslant-2.5$ \\
\hline Smoking & Admit to smoking or exhaled carbon monoxide $\geqslant 10 \mathrm{ppm}$ \\
\hline Sarcopenia & $\begin{array}{l}\text { Appendicular skeletal muscle mass index }<7.26 \mathrm{~kg} \cdot \mathrm{m}^{-2} \text { for males and } \\
<5.45 \mathrm{~kg} \cdot \mathrm{m}^{-2} \text { for females }\end{array}$ \\
\hline Inhaler device polypharmacy & Number of inhaler devices is $\geqslant 3$ \\
\hline $\begin{array}{l}\text { Inadequate inhaler device } \\
\text { technique }\end{array}$ & Inhaler technique rated as inadequate \\
\hline Nonadherence & Reported use of $<80 \%$ of prescribed treatment \\
\hline
\end{tabular}

$\mathrm{FEV}_{1}$ : forced expiratory volume in 1 s; FVC: forced vital capacity; 6MWT: 6-min walk test; PBS: Pharmaceutical Benefits Scheme; Hb: haemoglobin; hsCRP: high-sensitivity C-reactive protein; BMI: body mass index.

\section{Control}

Following the multidimensional assessment, the usual-care control group were treated according to best available evidence in the John Hunter Hospital severe asthma clinic [18]. The usual-care physician was blinded to the results of the multidimensional assessment. Usual care delivered in an established severe asthma clinic [18] included medical assessment by a respiratory physician. The participant's usual specialist physician provided the medical assessment, a review of comorbidities, an assessment of lung function and oxygen saturation by spirometry and pulse oximetry, and a diagnosis. The treating physician scheduled follow-up appointments as they deemed appropriate.

In addition, participants were reviewed by a nurse specialist in the severe asthma clinic. The role of the specialist nurse involved assessment and management of asthma management knowledge and skills, the provision of education, monitoring of adherence and implementation of adherence aiding strategies, trigger management and education for avoidance strategies including the provision of intensive smoking-cessation counselling. Moreover, the nurse monitored participants during treatment trials and administered add-on therapies. 


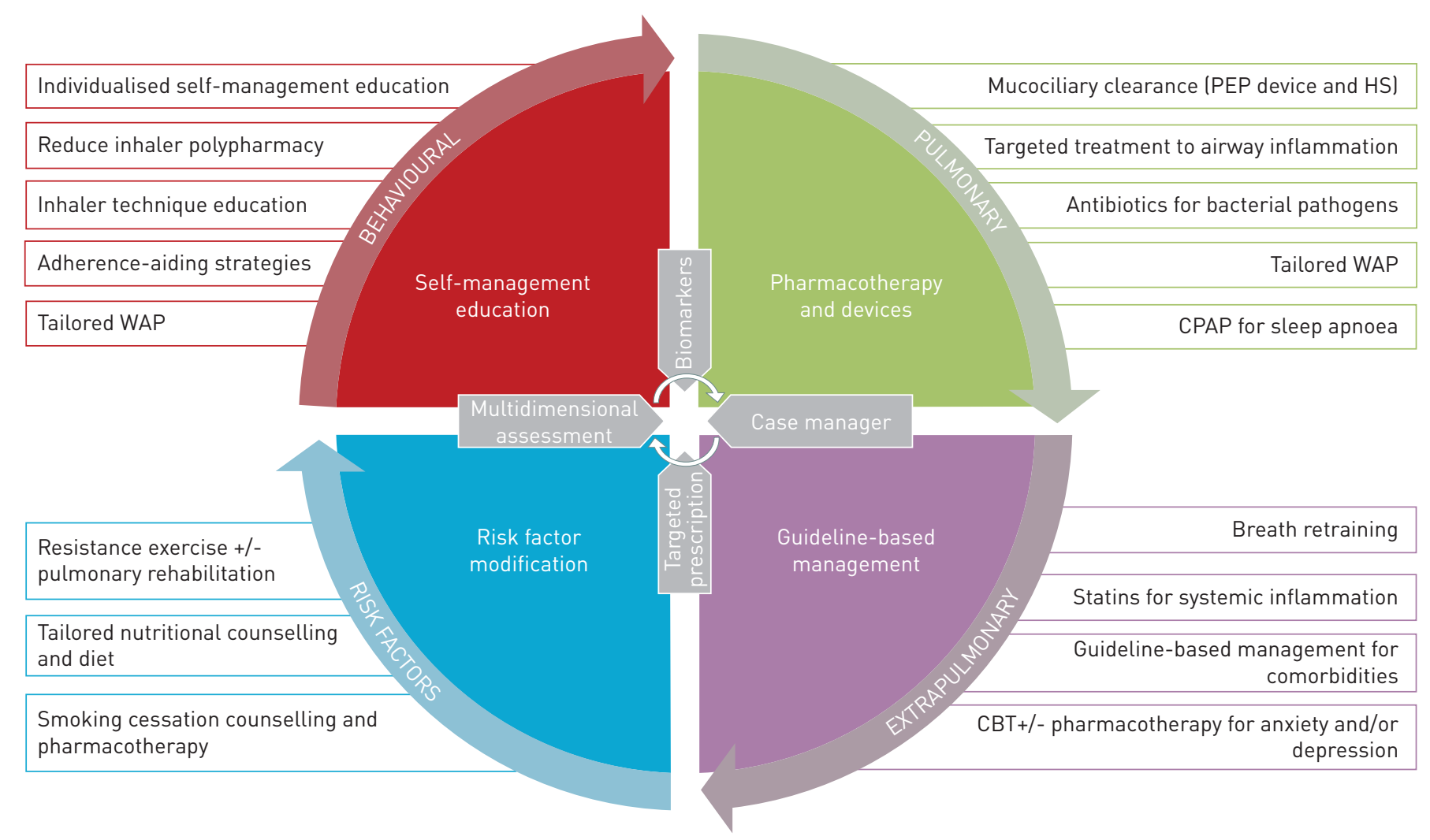

FIGURE 3 Components of the multidimensional assessment intervention. PEP: positive expiratory pressure; HS: hypertonic saline; WAP: written action plan; CPAP: continuous positive airway pressure; CBT: cognitive behavioural therapy.

Participants in the usual-care group also had access to a speech pathologist when appropriate, and other appropriate referrals were actioned from the usual-care severe asthma clinic.

\section{Outcome measures}

The primary outcome for the RCT was HRQoL measured by the Asthma Quality of Life Questionnaire (AQLQ) [21]. Secondary end-points included asthma control (Asthma Control Questionnaire (ACQ)-5 [22], exacerbations, airway and systemic inflammatory markers (hs-CRP) and health status (St George's Respiratory Questionnaire (SGRQ)) [23]. The outcomes were measured upon entry to the trial and at study completion.

\section{Sample size}

We anticipated a mean \pm SD change in AQLQ score of $0.8 \pm 0.8$ following the intervention. With $\alpha=0.05$ and power of $90 \%, 21$ participants were required in each group. Accounting for an expected attrition of $20 \%$, the randomisation target was 52 participants.

\section{Statistical analysis}

Data were analysed using SPSS (version 24; IBM, Chicago, IL, USA). For the cross-sectional assessment linear regression and Pearson's correlation were calculated. The RCT was analysed according to the intention-to-treat principle using generalised linear mixed models. Logistic regression, Poisson regression or negative binominal regression models were used as appropriate for binary and count data with baseline scores used as a covariate. To test the effect of the intervention component change scores were calculated by subtracting follow-up values from baseline values; the results are reported with $95 \%$ confidence intervals. Significance levels were set to $\mathrm{p}<0.05$.

\section{Results}

Multidimensional assessment of treatable traits

Overall, 140 participants were recruited to the cross-sectional study. The demographics and clinical characteristics of the cross-sectional participants are shown in table 2. There were no differences between clinical characteristics of the cross-sectional and RCT populations. We assessed 26 possible traits: nine 
TABLE 2 Baseline demographic and clinical characteristics for the cross-sectional study

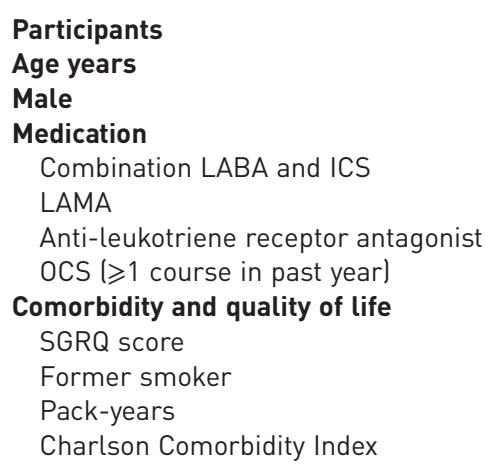

Data are presented as $\mathrm{n}$, mean $\pm \mathrm{SD}, \mathrm{n}(\%)$ or median (interquartile range). LABA: long-acting $\beta$-adrenoceptor agonist; ICS: inhaled corticosteroids; LAMA: long-acting muscarinic antagonist; OCS: oral corticosteroids; SGRQ: St George's Respiratory Questionnaire.

pulmonary, nine extrapulmonary and eight behavioural/risk factors (table 1). A mean \pm SD $10.44 \pm 3.03$ traits per participant were identified, comprising 3.01 \pm 1.54 pulmonary, $4.85 \pm 1.86$ extrapulmonary and $2.58 \pm 1.31$ behavioural/risk factors (figure 4a). The number of traits was significantly associated with health status (SGRQ) ( $\mathrm{r}=0.61, \mathrm{p}<0.001$ ), and for each additional trait identified there was a clinically and statistically significant decrement in SGRQ (4.02 units, $\mathrm{p}<0.001)$ (figure 4b).

RCT

We recruited 55 participants to the RCT; 28 were randomised to the intervention group and 27 to the control group. At trial completion, four intervention participants and two control participants either withdrew or were lost to follow-up (figure 1). Demographic characteristics for the RCT participants are displayed in table 3. The participants were predominantly female $(65.45 \%)$ and their mean \pm SD age was $52.23 \pm 13.86$ years. There were no differences between groups in the number of comorbidities or the traits identified using the multidimensional assessment (table 3).

The specific tailored treatments delivered as part of the intervention are presented in table 4 . The add-on pharmacotherapies prescribed to the control participants are described in supplementary table S5).
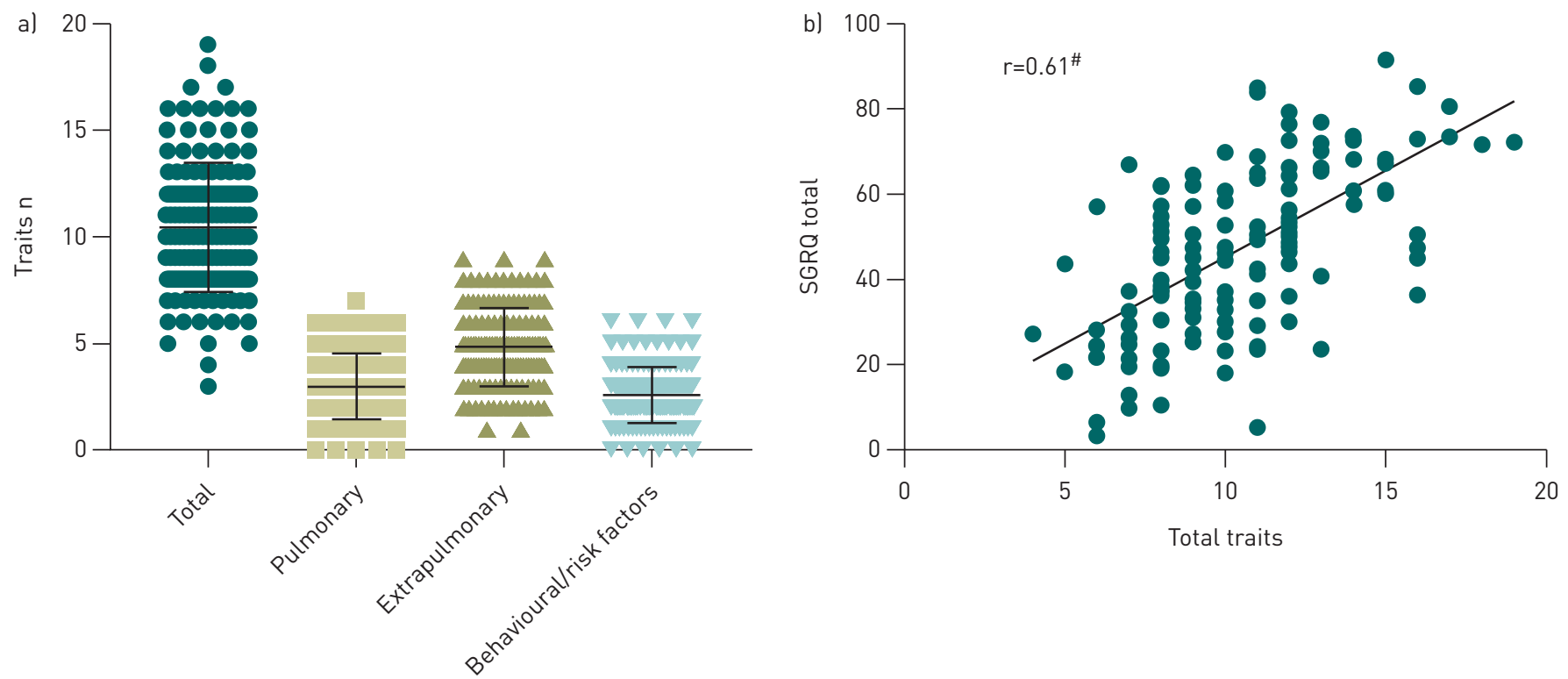

FIGURE 4 a) Number of total treatable traits, pulmonary traits, extrapulmonary traits and behavioural/risk factors for people with severe asthma in the cross-sectional study. Horizontal bars represent mean $\pm \mathrm{SD}$; b) correlation of treatable traits by health status (St George's Respiratory Questionnaire (SGRQ)) for people with severe asthma in the cross-sectional study. Diagonal line represents linear regression. ${ }^{\#}: p<0.0001$. 
TABLE 3 Baseline demographic and clinical characteristics for the randomised controlled trial

\begin{tabular}{|c|c|c|c|}
\hline & Overall & Intervention & Usual care \\
\hline Participants & 55 & 28 & 27 \\
\hline Male & 19 (34.54) & $10(35.71)$ & 9 (33.33) \\
\hline Age years & $52.23 \pm 13.86$ & $52.35 \pm 12.71$ & $52.09 \pm 15.21$ \\
\hline Pre-bronchodilator $\mathrm{FEV}_{1} \%$ pred & $71.11 \pm 21.41$ & $70.58 \pm 22.58$ & $71.65 \pm 20.59$ \\
\hline Post-bronchodilator FEV $1 \%$ pred & $73.21 \pm 21.29$ & $72.34 \pm 22.41$ & $74.07 \pm 20.50$ \\
\hline Post-bronchodilator FVC \% pred & $82.78 \pm 14.77$ & $81.32 \pm 15.61$ & $84.24 \pm 14.03$ \\
\hline Post-bronchodilator $\mathrm{FEV}_{1} / \mathrm{FVC}$ ratio & $68.62 \pm 13.37$ & $68.94 \pm 14.18$ & $68.29 \pm 12.77$ \\
\hline Former smoker & $29(53.71)$ & $15(53.56)$ & $14(51.85)$ \\
\hline Current smoker & $7(12.96)$ & $3(11.11)$ & $4(14.81)$ \\
\hline Smoking pack-years & $6.87 \pm 10.84$ & $6.50 \pm 10.50$ & $7.23 \pm 11.36$ \\
\hline $\mathrm{BMI} \mathrm{kg} \cdot \mathrm{m}^{-2}$ & $31.03(26.90-35.50)$ & $31.81(27.47-34.75)$ & $30.49(26.42-38.03)$ \\
\hline Number of comorbidities & $5.13 \pm 2.55$ & $4.96 \pm 2.36$ & $5.30 \pm 2.76$ \\
\hline Charlson Comorbidity Index & $0(0-1.00)$ & $0(0-2.00)$ & $0(0-0.25)$ \\
\hline \multicolumn{4}{|l|}{ Asthma control and quality of life } \\
\hline$A Q L Q$ & $4.72 \pm 1.34$ & $4.53 \pm 1.39$ & $4.91 \pm 1.28$ \\
\hline AQLQ symptoms & $4.48 \pm 1.38$ & $4.19 \pm 1.38$ & $4.77 \pm 1.34$ \\
\hline$A Q L Q$ environment & $4.83 \pm 1.66$ & $4.68 \pm 1.67$ & $4.99 \pm 1.65$ \\
\hline AQLQ emotions & $4.61 \pm 1.64$ & $4.54 \pm 1.69$ & $4.67 \pm 1.61$ \\
\hline AQLQ activity & $4.99 \pm 1.31$ & $4.85 \pm 1.40$ & $5.13 \pm 1.21$ \\
\hline SGRQ score & $49.07 \pm 21.01$ & $51.06 \pm 23.02$ & $47.08 \pm 19.02$ \\
\hline Asthma Control Questionnaire-5 & $2.04 \pm 1.20$ & $2.13 \pm 1.25$ & $1.94 \pm 1.18$ \\
\hline \multicolumn{4}{|l|}{ Biomarkers } \\
\hline $\mathrm{hsCRP} \mathrm{mg} \cdot \mathrm{mL}^{-1}$ & $3.40(1.87-8.42)$ & $3.90(2.10-9.60)$ & $3.30(1.40-8.10)$ \\
\hline Sputum neutrophils \% & 33.75 (16.50-62.25) & $32.00(15.87-64.13)$ & $36.25(18.00-60.25)$ \\
\hline Sputum eosinophils \% & 4.25 (1.00-13.75) & $3.50(0.75-14.50)$ & $6.00(1.00-13.75)$ \\
\hline$F_{\mathrm{eNO}} \mathrm{ppb}$ & $30.13 \pm 36.85$ & $28.96 \pm 39.64$ & $31.16 \pm 34.96$ \\
\hline \multicolumn{4}{|l|}{ Prescribed treatment } \\
\hline Combination LABA and ICS & $54(98.18)$ & $27(96.43)$ & $27(100)$ \\
\hline Beclomethasone-equivalent dose & 2000 (2000-2000) & 2000 (2000-2000) & $2000(2000-2000)$ \\
\hline LAMA & $20(38.46)$ & $8(30.76)$ & $12(46.15)$ \\
\hline Anti-leukotriene receptor antagonist & $6(10.90)$ & $3(10.71)$ & $3(11.11)$ \\
\hline
\end{tabular}

Data are presented as $\mathrm{n}, \mathrm{n}(\%)$, mean $\pm \mathrm{SD}$ or median (interquartile range). $\mathrm{FEV}_{1}$ : forced expiratory volume in $1 \mathrm{~s}$; FVC: forced vital capacity; BMI: body mass index; AQLQ: Asthma Quality of Life Questionnaire; SGRQ: St George's Respiratory Questionnaire; hsCRP: high-sensitivity C-reactive protein; $F_{\mathrm{eNO}}$ : fractional exhaled nitric oxide; LABA: long-acting $\beta$-adrenoceptor agonist; ICS: inhaled corticosteroid; LAMA: long-acting muscarinic antagonist.

The intervention group received treatment for $8.36 \pm 1.92$ traits during $5.32 \pm 2.53$ treatment visits over the 16-week period, compared to $2.37 \pm 2.32$ visits for the control group $(\mathrm{p}<0.001)$.

\section{Primary outcome}

The intervention led to a significant improvement in HRQoL (figure 5a). There was a significant between treatment group difference by time interaction for the AQLQ $(p<0.001)$. The AQLQ increased by a mean $(95 \%$ CI $) 0.86(0.49-1.23)$ units from baseline in the intervention group $(\mathrm{p}<0.001)$, compared to -0.004 $(-0.29-2.80)$ in the control group, indicating a clinically [24] and statistically significant improvement for the intervention group. There was no significant change in the usual-care control group (figure 5a). More participants in the intervention (55.56\%) achieved the minimal clinically important difference (MCID) of $\geqslant 0.5$ units compared to controls (22.22\%, $\mathrm{p}=0.02$; figure $5 \mathrm{~b}$ ), giving a number need to treat of 2.90 individuals. In the intervention group, AQLQ had a significant mean increase (improvement) from baseline to follow-up across all domains (symptoms 1.20, emotions 1.16 , environment 0.89 , activity 0.78 ; all $\mathrm{p}<0.001)$. There was no significant change in the usual-care group for any domain.

\section{Secondary outcomes}

There was a significant between-group difference in ACQ-5 $(p=0.009)$. In the intervention group the ACQ-5 decreased by a clinically important mean $(95 \% \mathrm{CI})-0.72(-1.25--0.18)$ units $(\mathrm{p}=0.011)$. There was no significant change in the usual-care group $0.08(-0.21-0.37 ; \mathrm{p}=0.557)$. There was a significantly higher proportion of intervention participants who achieved an ACQ-5 MCID of $>0.5$ units: $50.00 \%$ compared to $20.83 \%(\mathrm{p}=0.034)$. 
TABLE 4 Traits treated in the intervention group and reponse to treatment

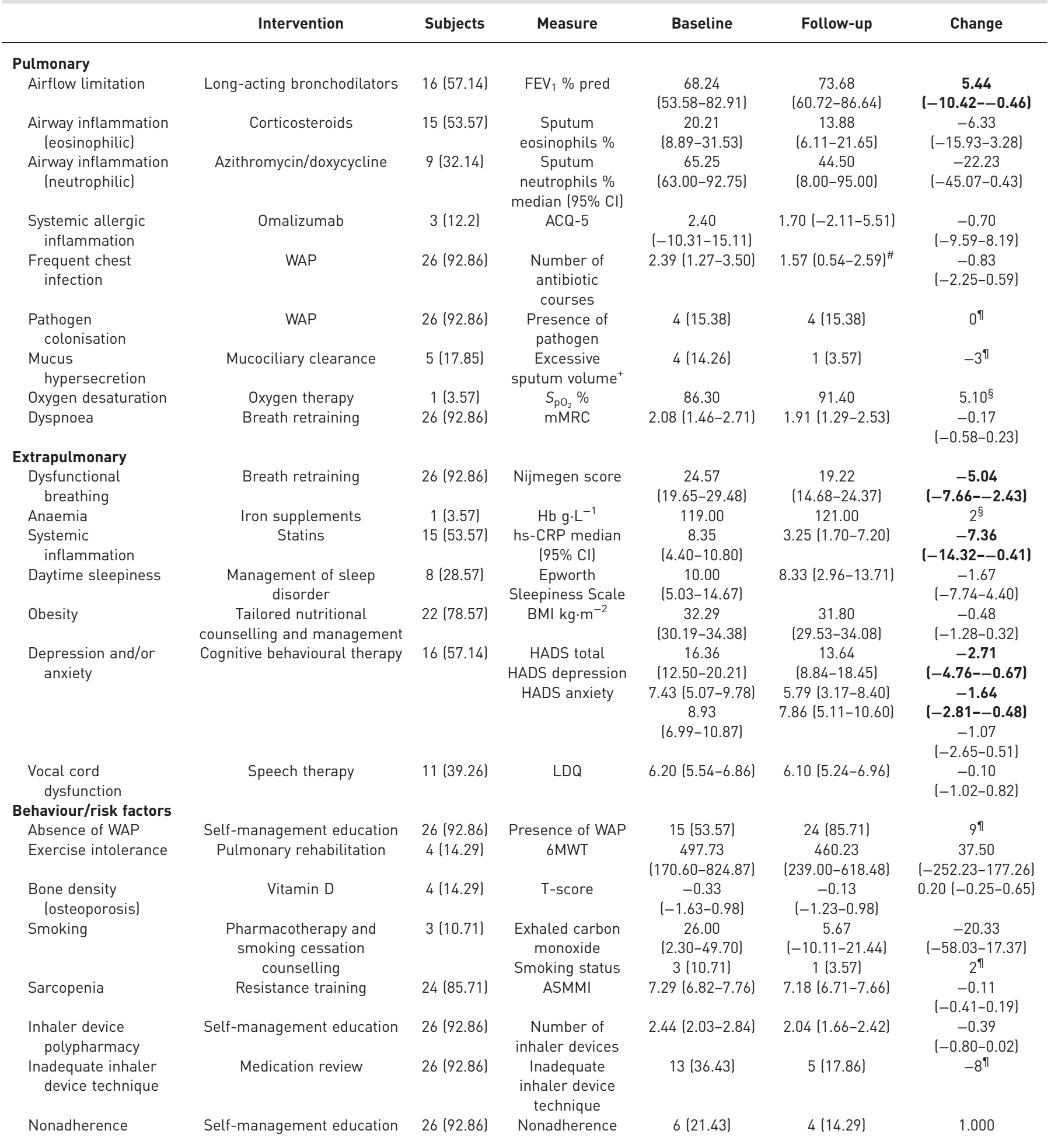

Data are presented as $\mathrm{n}(\%)$ or mean $(95 \% \mathrm{Cl})$, unless otherwise stated. Includes only individuals who receive the specific individualised interventions. Baseline and follow-up scores, and change scores based on marginal means. Bold type indicates that change score confidence intervals do not overlap zero. $\mathrm{FEV}_{1}$ : forced expiratory volume in $1 \mathrm{~s}$; $\mathrm{ACQ}$ : Asthma Control Questionnaire; $\mathrm{S}_{\mathrm{pO}_{2}}$ : peripheral oxygen saturation; mMRC: modified Medical Research Council; Hb: haemoglobin; hsCRP: high-sensitivity C-reactive protein; BMI: body mass index; HADS: Hospital Anxiety and Depression Scale; LDQ: Laryngeal Dysfunction Questionnaire; WAP: written action plan; 6MWT: 6-min walk test; ASMMI: appendicular skeletal muscle mass. ${ }^{\#}$ : converted to annualised rate; ${ }^{\text {१: }}$ number of people who changed after the intervention; ${ }^{+}$: a volume $\geqslant 25 \mathrm{~mL}$ of mucus produced daily for the past week in the absence of infection; ${ }^{\S}$ : intervention only received by $n=1$, confidence interval not calculated. 
a)

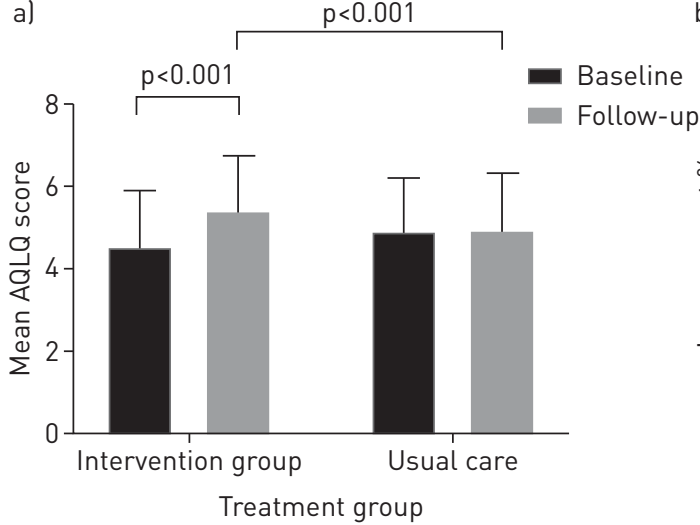

b)

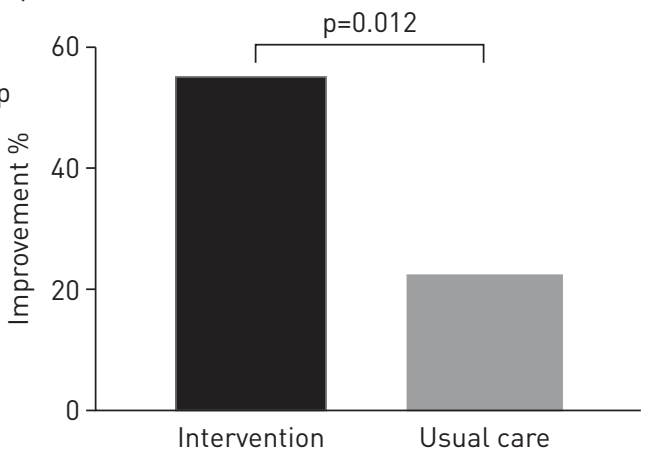

FIGURE 5 a) Effect of interventions on asthma quality of life; b) responder analysis for the mean clinical important difference improvement in asthma quality of life for the intervention group and the control group. Horizontal bars represent standard deviations. AQLQ: Asthma Quality of Life Questionnaire.

Health status was also measured using the SGRQ and improved significantly in the intervention group, but not in the control group. The mean (95\% CI) change in SGRQ for the intervention group was -13.42 $(-18.44--8.39)$ units versus a $-2.55(-6.72-1.63)$ unit $(\mathrm{p}=0.001)$ difference in the control group.

The change scores and confidence intervals for other secondary outcomes by treatment condition at baseline and follow-up are presented in supplementary table S6.

\section{Impact of targeted therapies}

While the study was not powered to detect differences in the individualised interventions, forced expiratory volume in $1 \mathrm{~s}$ improved in those who received a medication review and inhaled corticosteroid dose adjustment. Nijmegen score improved in those who received breath retraining, hsCRP improved in the statin-treated participants and depression scores improved with targeted cognitive behavioural therapy. In addition, inhaler technique improved. There were clinically important reductions in the sputum neutrophil and eosinophil percentages in those who received targeted treatments, but the confidence interval for eosinophil count did overlap zero (table 4), probably due to the small sample in these groups.

Similarly, the study was not powered to detect differences in exacerbations, but there were noticeable trends for this outcome, with an incidence rate ratio favouring the intervention for all exacerbation types (supplementary table S7). This was statistically significant for primary-care presentations for acute asthma (control group rate 0.50 and intervention group 0.07; incidence rate ratio $0.14,95 \%$ CI $0.02-0.93$ ).

\section{Adverse events}

Intervention-group adverse events included a possible allergic reaction to the meal replacements $(\mathrm{n}=1)$, a fall at home $(n=1)$, dizziness post-sputum induction $(n=1)$, minor complication post-venepuncture $(n=1)$, infection post-laryngoscopy $(n=1)$, sore muscles post-exercise $(n=4)$ and skin irritation from nicotine patch $(n=1)$. In the control group one participant had a minor complication post-venepuncture.

\section{Discussion}

People with severe asthma require improved management strategies $[6,8,25]$. We report the results of a personalised medicine approach to severe asthma where we show that multidimensional assessment can identify the many treatable traits that exist in this disease, and that targeting treatments to these traits leads to significant improvements in HRQoL, asthma control and asthma attacks. To our knowledge this is the first RCT to test a multidimensional and targeted approach to severe asthma, and the first treatable-traits RCT to be reported. This work advances knowledge of personalised disease-management strategies for this challenging airway disease, and provides the much needed data to support recently proposed new treatment paradigms $[5,11,13,14,26]$.

There have been many calls to rethink the approach to complex airway diseases $[5,11,13,14,25,27]$; however, until now there have been no RCTs evaluating the efficacy of a multidimensional assessment followed by personalised targeted treatments. A meta-analysis of severe asthma multidimensional assessment showed improved asthma control, HRQoL and reduced exacerbations and recommended that RCTs were needed [16]. Similar conclusions were reached by the Lancet commission [5], and others [26]. The application of personalised medicine in airway diseases can be achieved using an approach that 
carefully evaluates the treatable traits [13] and applies therapy that is precisely tailored to the specific disease traits that manifest in each individual [13,17]. Clinical trials of inflammometry in asthma and COPD emerged in the early 2000s, where individuals with eosinophilic airway inflammation were identified and treatment implemented to target this inflammatory endotype, leading to reduced exacerbations [28-30]. Monoclonal antibody therapies targeting type-2 high inflammation in asthma have further progressed this area [31]. These studies support a personalised-medicine paradigm where inflammatory endotypes are identified and treatment is targeted to specific pathways [26]. We applied this approach to the eosinophilic inflammatory endotype, and additionally included macrolide antibiotics to target the neutrophilic endotype [32,33] and statins for systemic inflammation [17], in addition to targeting comorbidities and risk factors. It is important to note that our study predated the availability of anti-interleukin-5 therapy in Australia. As such, we used oral corticosteroids (OCS) to target eosinophilic airway inflammation. We recognise the need to adapt our algorithm to include currently available and effective treatments that target eosinophilic disease without the toxicity of OCS [34]. However, the use of OCS provides important knowledge in terms of proof of concept for this trial.

Additionally, we recognise that personalised medicine extends beyond one pathway [26], and is an approach where treatments are targeted to the needs of individuals, not only on the basis of biomarkers, but also genetic, phenotypic or psychosocial characteristics that distinguish the individual [35]. Hence, approaches to complex airway diseases need to consider these factors, especially since individuals with severe asthma express multiple treatable traits [26, 36]. This RCT reports the success of this holistic approach. In our study, quality of life improved to a greater degree than that seen in recent studies of monoclonal antibodies and macrolide antibiotics [34], suggesting that the multidimensional intervention which targets traits in each domain is multiplicative, and treatment beyond pharmacotherapy is necessary. We propose that treating the multiple manifestations has an additional stacked effect and this is one of the major benefits of the treatable-traits approach.

The patient-reported outcome of HRQoL was the primary end-point. The impact of targeted treatment on this outcome was large. This is consistent with a proof-of-concept controlled study of a similar intervention in COPD [17]. In that study SGRQ was the primary outcome and improved by 14 units; the SGRQ in our current study improved by a similar magnitude of 13.4 units, supporting the reproducibility of the intervention and the results. Severe asthma has a major impact on health status that results in long-term and devastating burdens $[6,8,25]$. Therefore, improving health status is an important goal from a patient perspective, and which has not yet been achieved despite advances in pharmacotherapy [37]. This was an appropriate outcome for this study, as the measure assesses multiple domains, which was necessary for the multicomponent intervention tested. Interestingly and surprisingly, we did not observe a change in health status in the control group, which involved management in an established severe asthma clinic. Several factors may have influenced this. First, the usual-care group did not include a case manager to assist with implementation of the treatment plan; this likely resulted in the control participants receiving fewer treatments over the course of the intervention period. Despite this, the usual-care group were treated by an expert multidisciplinary team. Second, the intervention was delivered as a simultaneous approach compared to the sequential approach of the severe asthma clinic [38]; as such the intensity of the treatment in the intervention was greater. However, this supports the approach proposed, as the right patients receive the right treatments within an efficient time period. Finally, the intervention involved an algorithm for treatment of inflammatory endotypes, which all intervention participants received, as opposed to a more ad hoc approach in the control group [18]. We advocate that the magnitude of the effect is achieved by not one individual aspect of the programme, but the "stacking" or additive effects of multiple treatment interventions [17].

Asthma attacks are another important outcome in severe asthma [36, 39]. We demonstrated significantly fewer unscheduled primary-care presentations for acute asthma in the intervention group. For other acute asthma events there were trends toward fewer attacks. Our follow-up period was only 4 months, which was probably not long enough to evaluate the impact of the intervention on exacerbations, and nor was the study powered for this outcome. Nonetheless, these results are promising, and should be addressed in future studies.

We report the results of a multidimensional assessment in a large group of severe asthma patients. We show that multidimensional assessment can be used to identify the prevalence of these treatable traits, and demonstrate the significant trait burden experienced by severe asthma patients. Furthermore, the number of traits were significantly correlated with health status and each additional trait identified was associated with a clinically significant decrement in SGRQ, a result we have also reported in COPD [15]. In the absence of a multidimensional assessment many of these important clinical characteristics may remain unrecognised, and therefore untreated, as is reported in a systematic review of severe asthma assessment [16]. The traits we included in the assessment and individualised interventions were comprehensive, but 
not exhaustive. Identifying traits that have the biggest impact on outcomes whether that be exacerbations or quality of life is important for the design of future treatable trait trials and for implementation into practice [11]. We have previously reported traits that predict future exacerbation in severe asthma, but more work is needed in this area [36].

Another important finding from a disease-management and self-efficacy perspective, was that there were suboptimal self-management traits in patients with severe asthma. This suggests that the basic management strategies and goals are not adequately achieved as part of usual disease management. Suboptimal self-management skills are associated with increased risk of attacks in severe asthma [36], and our intervention ensured the assessment of these traits and applied individualised treatments to improve them. An important component was the use of a case manager in the delivery of the intervention. This was a key difference from the usual-care severe asthma clinic [18], and suggests the importance of this role in the delivery of complex and multicomponent interventions [39].

Our RCT was a complex intervention and provides novel insight for the design of future studies examining multidimensional assessment and individualised management. Our approach was comprehensive and targeted all of the traits identified. The intervention group did receive more treatment visits than the control group. We did not seek to define how many visits the control group received, as we sought to replicate usual care within a severe asthma clinic. Furthermore, we sought to perform a pragmatic RCT, comparing our intervention to current best practice. However, we do acknowledge that this could potentially impact on the results. Nevertheless, we believe that this is an important consideration for future implementation studies. It is likely that this complex population will require more intensive intervention than current practice allows. This is an important observation that needs to be recognised. We propose that healthcare systems need to re-evaluate care for individuals with high healthcare needs [14]. Yet, whether an approach that included fewer traits would have led to the same effect is not known, but is worth considering.

While the provision of this intervention resulted in improved outcomes for patients with severe asthma, it recognisably comes with an increased economic cost. A health-economic evaluation is required in future work. We hypothesise that the delivery of personalised medicine would become cost-neutral or even result in cost savings by reducing the iatrogenic consequences of toxic treatment and the cost of asthma attacks and burden on the acute healthcare sector. Economic modelling suggests that the direct healthcare cost savings that would flow from better targeted treatment for asthma in Australia ranges from AUD 129 million to AUD 1288 million [40].

Our study has a number of strengths and some limitations. In terms of our RCT, the sample size is relatively small, and the intervention and control groups were not matched for contact time. This means that larger trials are needed in order to inform practice and implementation of such an approach. Still, we urge the importance of these data particularly when evaluating such complex and multicomponent interventions [41], and therefore argue the necessity of these data in progressing this important area of airway disease management. Furthermore, the follow-up time was only 4 months; future trials should include a longer follow-up to assess the impact of interventions that require significant behaviour change, for example adherence, diet and physical activity and of course the evaluation of exacerbation of time.

\section{Conclusions}

Multidimensional assessment and targeted management of severe asthma allows for the detection of treatable traits that can be targeted using a personalised medicine approach. This approach significantly improves health status in people with severe asthma and represents a new approach to this complex disease.

Acknowledgements: The authors would like to acknowledge the participants enrolled in the study. We also acknowledge the clinical research team involved in the intervention delivery and outcome assessment, including Kelly Steel (John Hunter Hospital, Newcastle, Australia), Amber Smith, Penny Baines and Michelle Rostas (University of Newcastle, Newcastle, Australia), and the laboratory processing team at the Priority Research Centre for Healthy Lungs. Finally, we acknowledge Heather Powell (John Hunter Hospital) for managing the randomisation schedule.

Author contributions: V.M. McDonald conceived and executed the study, supervised the conduct of the study, interpreted data and wrote the manuscript. V.L. Clark performed the data analysis, interpreted data, contributed to preparation of and critically reviewed the manuscript. L. Cordova-Rivera collected data and edited and critically reviewed the manuscript. P.A.B. Wark contributed to the design of the study, provided medical support and edited and critically reviewed the manuscript. K.J. Baines supervised the conduct of laboratory aspects of the study and critically reviewed the manuscript. P.G. Gibson conceived and executed the study, supervised the study, interpreted data and contributed to the preparation of and critically reviewed the manuscript. 
Conflict of interest: V.L. Clark reports personal fees for research and providing educations from AstraZeneca, and receives a fellowship from the National Health and Medical Research Council, Centre of Research Excellence in Severe Asthma. L. Cordova-Rivera has nothing to disclose. P.A.B. Wark has nothing to disclose. K.J. Baines reports grants from Hunter Medical Research Institute, during the conduct of the study. P.G. Gibson reports personal fees for lectures from AstraZeneca, GlaxoSmithKline and Novartis, grants from AstraZeneca and GlaxoSmithKline, outside the submitted work. V.M. McDonald reports grants from Hunter Medical Research Institute, National Health and Medical Research Council and John Hunter Hospital Charitable Trust Research Grants, during the conduct of the study; grants and personal fees for educational lectures from GSK and AstraZeneca, outside the submitted work.

Support statement: This work was supported by the National Health and Medical Research Council, Hunter Medical Research Institute, The University of Newcastle, The John Hunter Hospital Charitable Trust. Funding information for this article has been deposited with the Crossref Funder Registry.

\section{References}

1 Chung KF, Wenzel SE, Brozek JL, et al. International ERS/ATS guidelines on definition, evaluation and treatment of severe asthma. Eur Respir J 2014; 43: 343-373.

2 Gibson PG, McDonald VM. Management of severe asthma: targeting the airways, comorbidities and risk factors. Intern Med J 2017; 47: 623-631.

3 Braman SS. The global burden of asthma. Chest 2006; 130: 1 Suppl., 4S-12S.

4 Hekking PP, Wener RR, Amelink M, et al. The prevalence of severe refractory asthma. J Allergy Clin Immunol 2015; 135: 896-902.

5 Pavord ID, Beasley R, Agusti A, et al. After asthma: redefining airways diseases. Lancet 2018; 391: 350-400.

6 Foster JM, McDonald VM, Guo M, et al. "I have lost in every facet of my life": the hidden burden of severe asthma. Eur Respir J 2017; 50: 1700765.

7 Chronic Respiratory Diseases in Australia. Canberra, Australian Institute of Health and Welfare, 2005.

8 McDonald VM, Hiles SA, Jones KA, et al. Health-related quality of life burden in severe asthma. Med J Aust 2018; 209: S28-S33.

9 Sweeney J, Brightling CE, Menzies-Gow A, et al. Clinical management and outcome of refractory asthma in the UK from the British Thoracic Society Difficult Asthma Registry. Thorax 2012; 67: 754-756.

10 Royal College of Physicians. Why Asthma Still Kills: the National Review of Asthma Deaths (NRAD) Confidential Enquiry Report. London, Royal College of Physicians, 2014.

11 McDonald VM, Fingleton J, Agusti A, et al. Treatable traits: a new paradigm for 21st century management of chronic airway diseases. Eur Respir J 2019; 53: 1802058

12 Ebmeier S, Thayabaran D, Braithwaite I, et al. Trends in international asthma mortality: analysis of data from the WHO Mortality Database from 46 countries (1993-2012). Lancet 2017; 390: 935-945.

13 Agusti A, Bel E, Thomas M, et al. Treatable traits: toward precision medicine of chronic airway diseases. Eur Respir J 2016; 47: 410-419.

14 Gibson PG, McDonald VM, Marks GB. Asthma in older adults. Lancet 2010; 376: 803-813.

15 McDonald VM, Simpson JL, Higgins I, et al. Multidimensional assessment of older people with asthma and COPD: clinical management and health status. Age Ageing 2011; 40: 42-49.

16 Clark VL, Gibson PG, Genn G, et al. Multidimensional assessment of severe asthma: a systematic review and meta-analysis. Respirology 2017; 22: 1262-1275.

17 McDonald VM, Higgins I, Wood LG, et al. Multidimensional assessment and tailored interventions for COPD: respiratory utopia or common sense? Thorax 2013; 68: 691-694.

18 McDonald VM, Vertigan AE, Gibson PG. How to set up a severe asthma service. Respirology 2011; 16: 900-911.

19 Treasure T, MacRae KD. Minimisation: the platinum standard for trials? Randomisation doesn't guarantee similarity of groups; minimisation does. BMJ 1998; 317: 362-363.

20 McDonald VM, Higgins I, Simpson JL, et al. The importance of clinical management problems in older people with COPD and asthma: do patients and physicians agree? Prim Care Respir J 2011; 20: 389-395.

21 Juniper EF, Guyatt GH, Ferrie PJ, et al. Measuring quality of life in asthma. Am Rev Respir Dis 1993; 147: 832-838.

22 Juniper EF, O'Byrne PM, Guyatt GH, et al. Development and validation of a questionnaire to measure asthma control. Eur Respir J 1999; 14: 902-907.

23 Jones PW. St George's Respiratory Questionnaire: MCID. COPD 2005; 2: 75-79.

24 Juniper GG, Willan A, Griffith LE. Determining a minimal important change in a disease-specific Quality of Life Questionnaire. J Clin Epidemiol 1994; 47: 81-87.

25 McDonald VM, Kennington E, Hyland M. Understanding the experience of people living with severe asthma. In: Chung KF, Israel E, Gibson PG, eds. Severe Asthma (ERS Monograph). Sheffield, European Respiratory Society, 2019; pp. 16-29.

26 Agusti A, Bafadhel M, Beasley R, et al. Precision medicine in airway diseases: moving to clinical practice. Eur Respir J 2017; 50: 1701655.

27 Woodruff PG, Agusti A, Roche N, et al. Current concepts in targeting chronic obstructive pulmonary disease pharmacotherapy: making progress towards personalised management. Lancet 2015; 385: 1789-1798.

28 Green RH, Brightling CE, McKenna S, et al. Asthma exacerbations and sputum eosinophil counts: a randomised controlled trial. Lancet 2002; 360: 1715-1721.

29 Jayaram L, Pizzichini MM, Cook RJ, et al. Determining asthma treatment by monitoring sputum cell counts: effect on exacerbations. Eur Respir J 2006; 27: 483-494.

30 Siva R, Green RH, Brightling CE, et al. Eosinophilic airway inflammation and exacerbations of COPD: a randomised controlled trial. Eur Respir J 2007; 29: 906-913.

31 Grainge CL, Maltby S, Gibson PG, et al. Targeted therapeutics for severe refractory asthma: monoclonal antibodies. Expert Rev Clin Pharmacol 2016; 9: 927-941. 
32 Gibson PG, Yang IA, Upham JW, et al. Effect of azithromycin on asthma exacerbations and quality of life in adults with persistent uncontrolled asthma (AMAZES): a randomised, double-blind, placebo-controlled trial. Lancet 2017; 390: 659-668.

33 Simpson JL, Powell H, Boyle MJ, et al. Clarithromycin targets neutrophilic airway inflammation in refractory asthma. Am J Respir Crit Care Med 2008; 177: 148-155.

34 Holguin F, Cardet JC, Chung KF, et al. Management of severe asthma: a European Respiratory Society/American Thoracic Society Guideline. Eur Respir J 2020; 55: 1900588.

35 Jameson JL, Longo DL. Precision medicine - personalized, problematic, and promising. N Engl J Med 2015; 372: 2229-2234.

36 McDonald VM, Hiles SA, Godbout $\mathrm{K}$, et al. Treatable traits can be identified in a severe asthma registry and predict future exacerbations. Respirology 2019; 24: 37-47.

37 Katsaounou P, Odemyr M, Spranger O, et al. Still fighting for breath: a patient survey of the challenges and impact of severe asthma. ERJ Open Res 2018; 4: 00076-2018.

38 Spring B, Schneider K, McFadden HG, et al. Multiple behavior changes in diet and activity: a randomized controlled trial using mobile technology. Arch Intern Med 2012; 172: 789-796.

39 McDonald VM, Gibson PG. Exacerbations of severe asthma. Clin Exp Allergy 2012; 42: 670-677.

40 Gibson PG, Marks G, Sly P, et al. Case for action- proposal to NHMRC. Targeted therapy for asthma. Date last updated: February 2015. https://www.nhmrc.gov.au/file/6121/download?token=2gzhvJ3H

41 Craig P, Dieppe P, Macintyre S, et al. Developing and Evaluating Complex Interventions. London, Medical Research Council, 2006. 